\title{
Barium sulfate crystallization in synthetic seawater
}

\author{
Matthew Boon, Franca Jones*
}

Department of Chemistry, Nanochemistry Research Insitute, Curtin University GPO Box U1987 WA 6845

*Corresponding author, email : F.Jones@curtin.edu.au phone: (618) 92667677 fax: (618) 92662300

\section{Supplementary information}
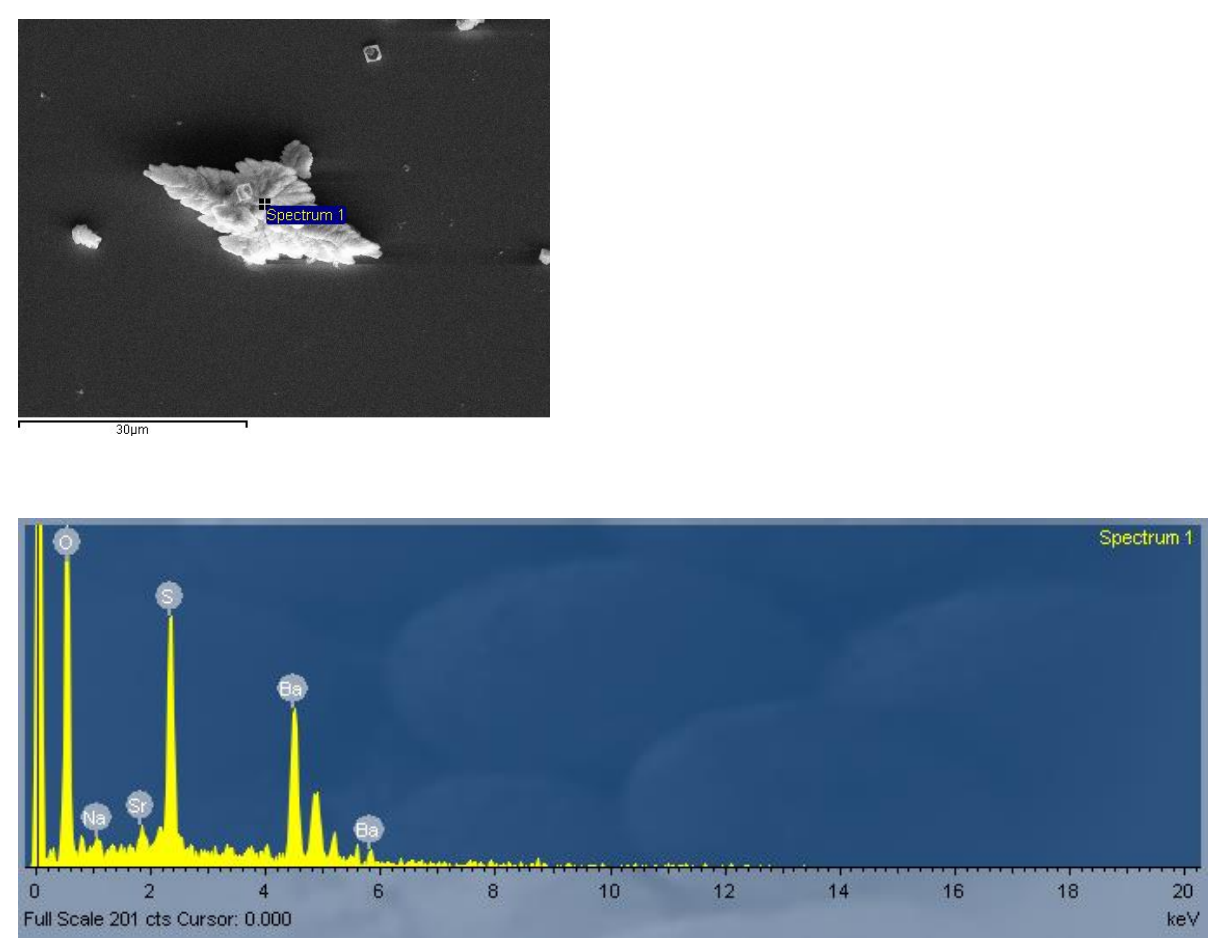

SFigure 1. SEM of dendritic barite particle formed in the presence of SSW and the EDS spectrum in the position shown 


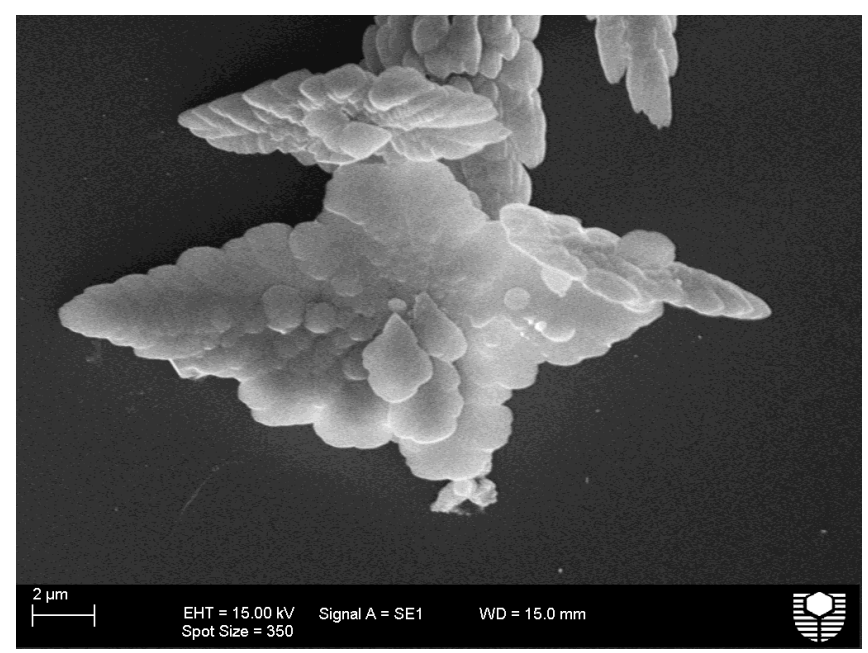

SFigure 2. SEM image of barium sulfate formed in $\mathrm{SSW}$ at $\mathrm{pH} 8$

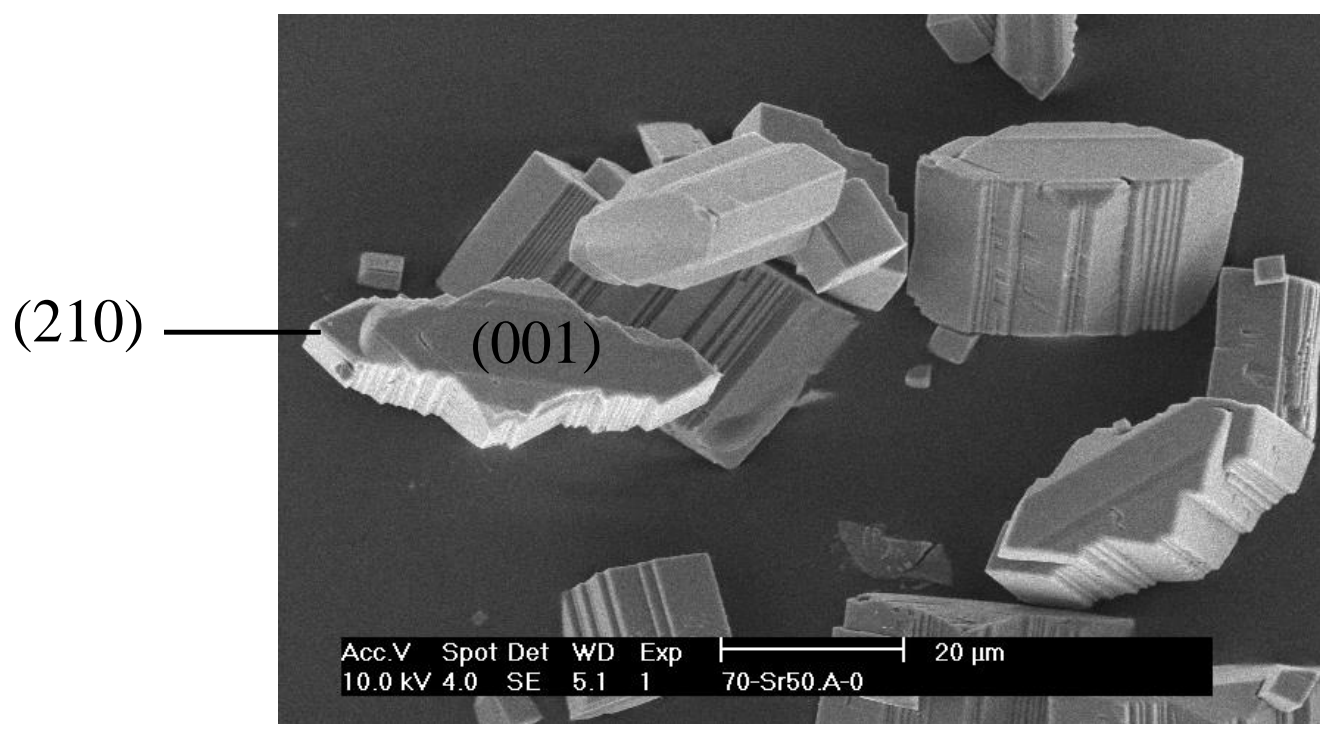

SFigure 3. SEM image of strontium sulfate grown at $70{ }^{\circ} \mathrm{C}$. (with permission from Wannapa Boonwannich) 


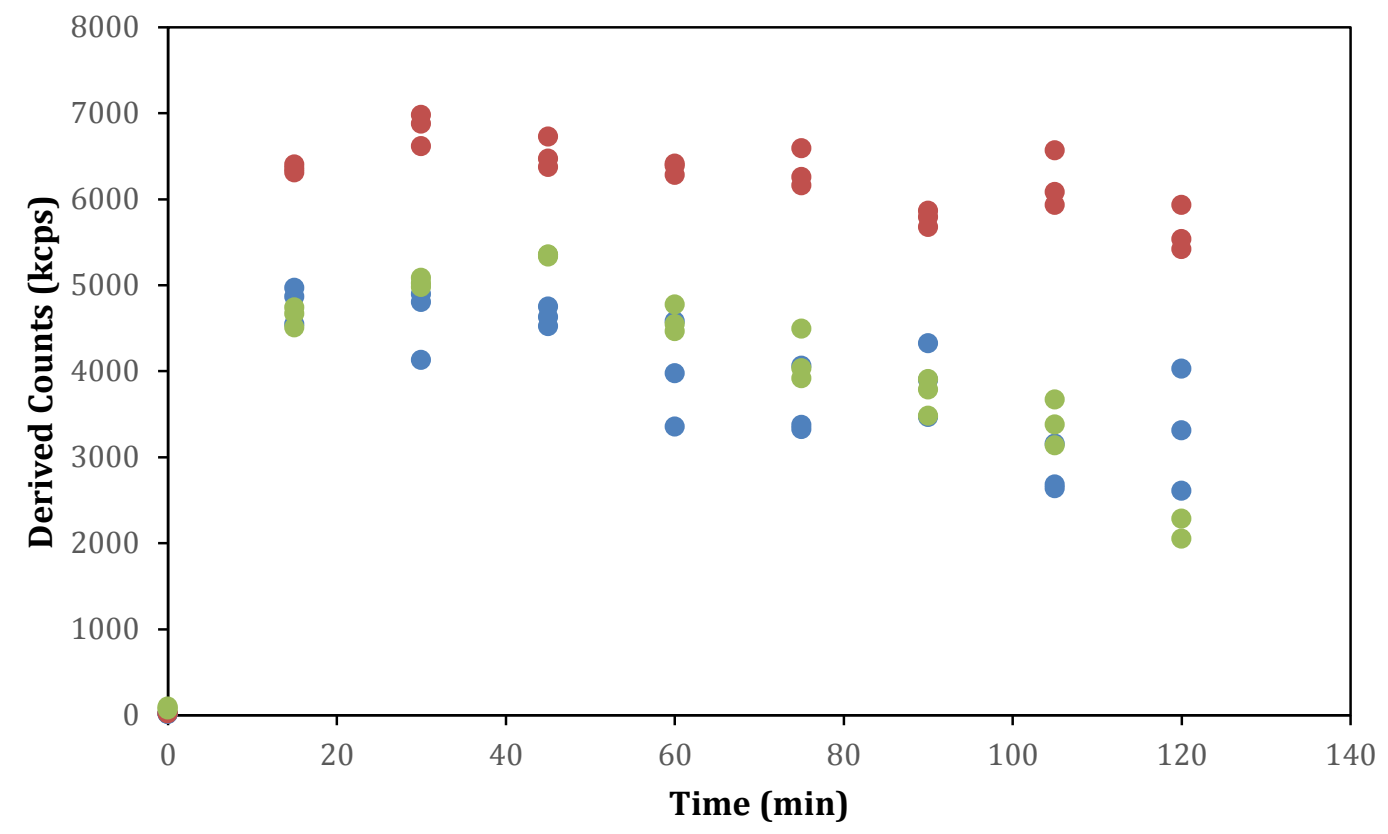

SFigure 4. DLS data when different stirring rate is used (red circle) and results where same speed is used (blue, green circles) for barium sulfate formed at $\mathrm{pH} 8$ and 1:1 ion ratio

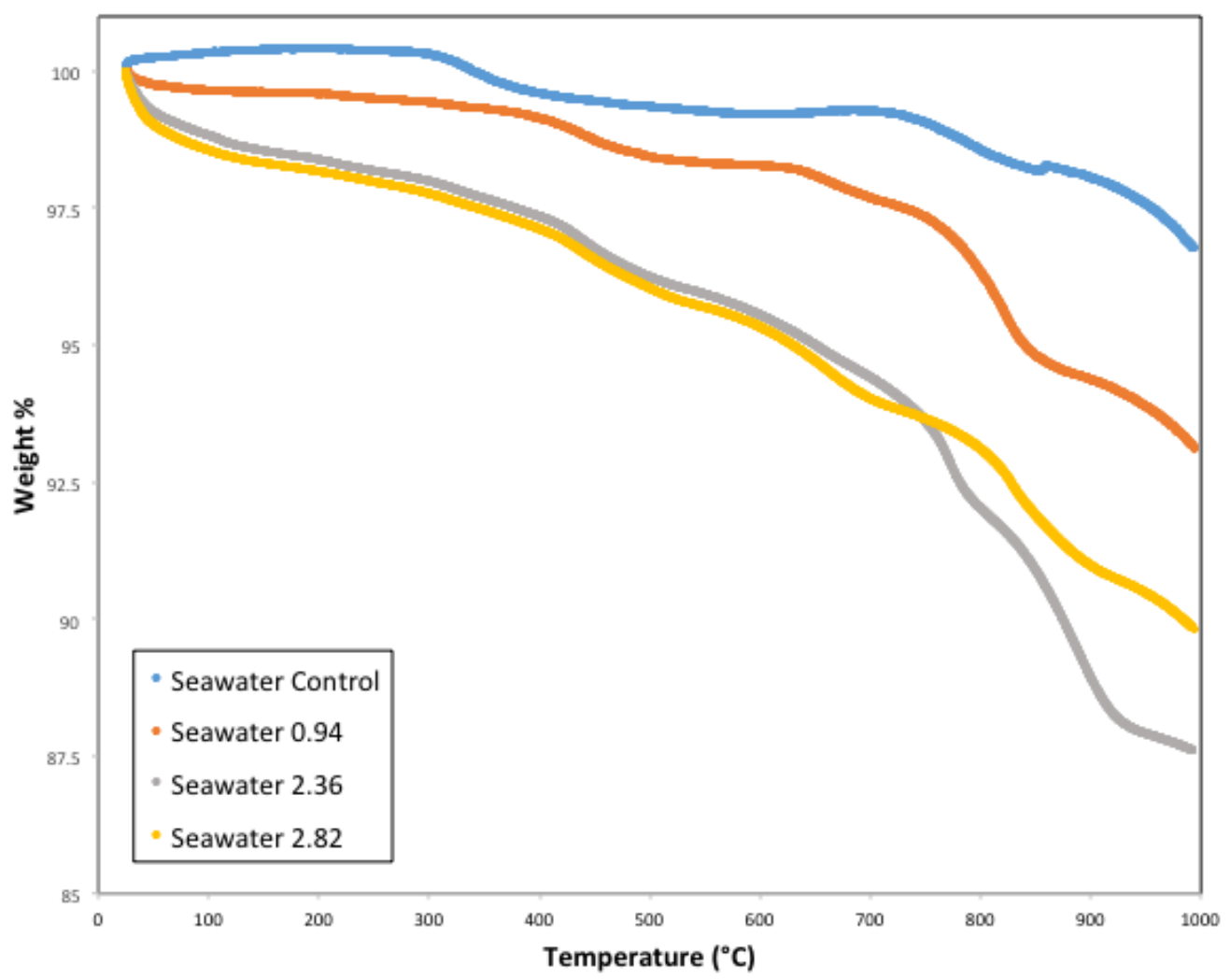

SFigure 5. TGA results for barium sulfate formed in the presence of SSW and at different silicate concentration 


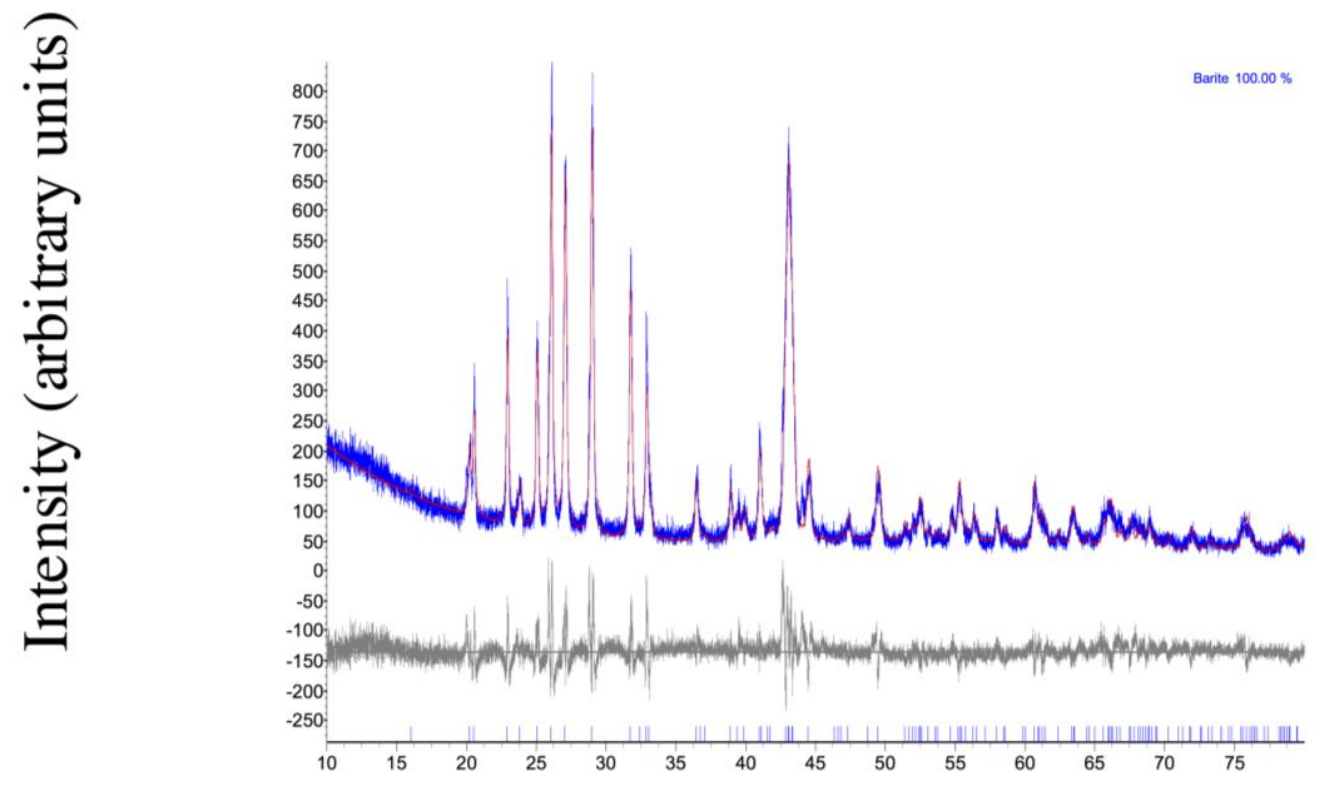

\section{2theta}

SFigure 6. XRD pattern of barium sulfate formed in SSW (blue trace) and the Rietveld refined pattern (red trace). The difference pattern is shown in grey. 

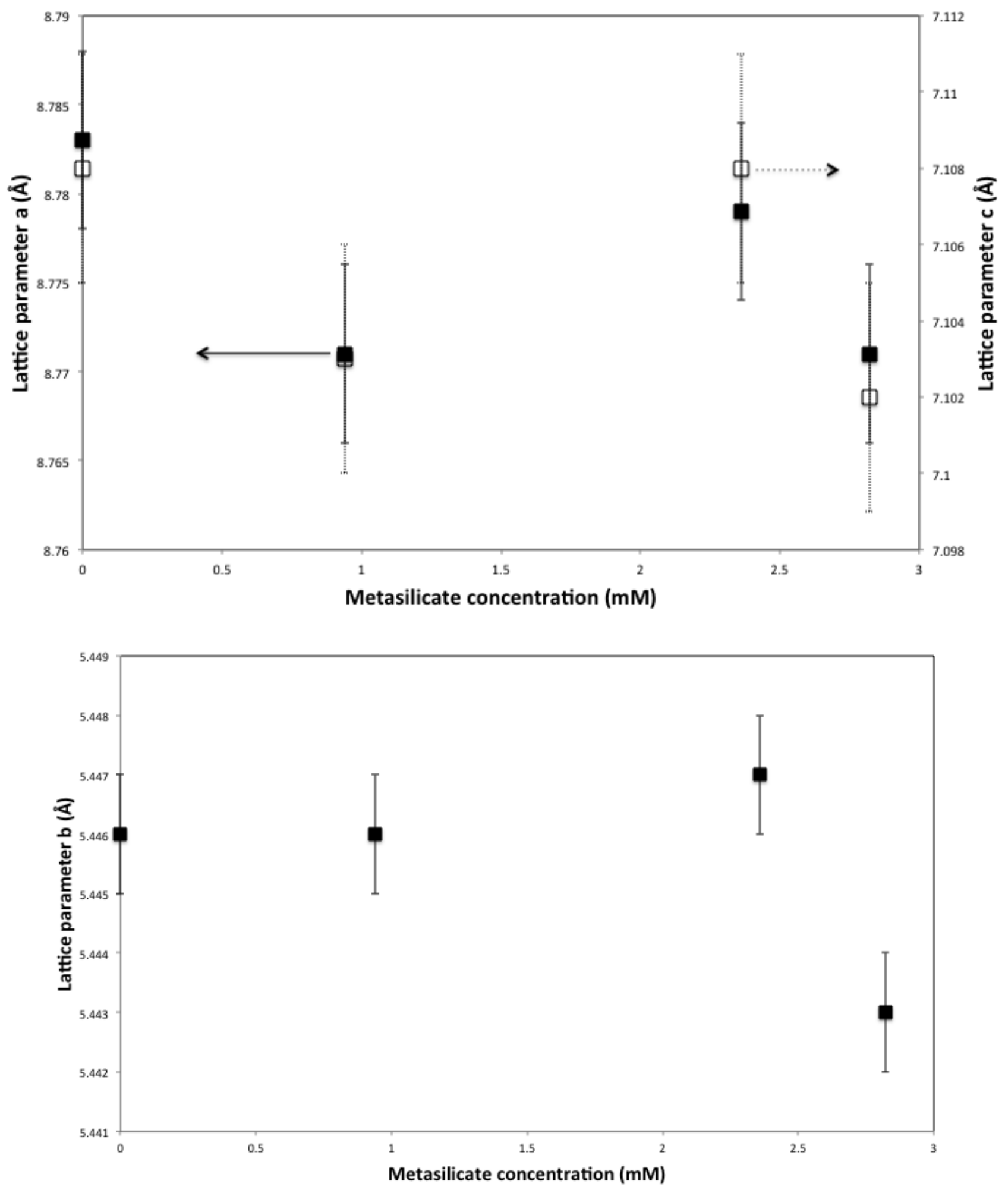

SFigure 7. Change in the (a) $a$ and $c$ lattice parameter and (b) $b$ lattice parameter as determined from Rietveld refinement versus silicate concentration for barium sulfate formed in SSW and different silicate concentrations 


\section{Simulation of Strontium substitution and expected XRD pattern}

Note, the supercell size has been used, meaning the single unit cell lattice parameter changes are actually smaller. The different colours represent whether barium ions substituted into the celestite structure (filled circles) or whether strontium ions substituted into the barite structure (open circles). Different substitution mechanisms were considered; namely whether the $\mathrm{Sr}^{2+}$ ions were grouped closely or dispersed throughout the structure and this is shown by multiple points for the same $\%$ substitution.
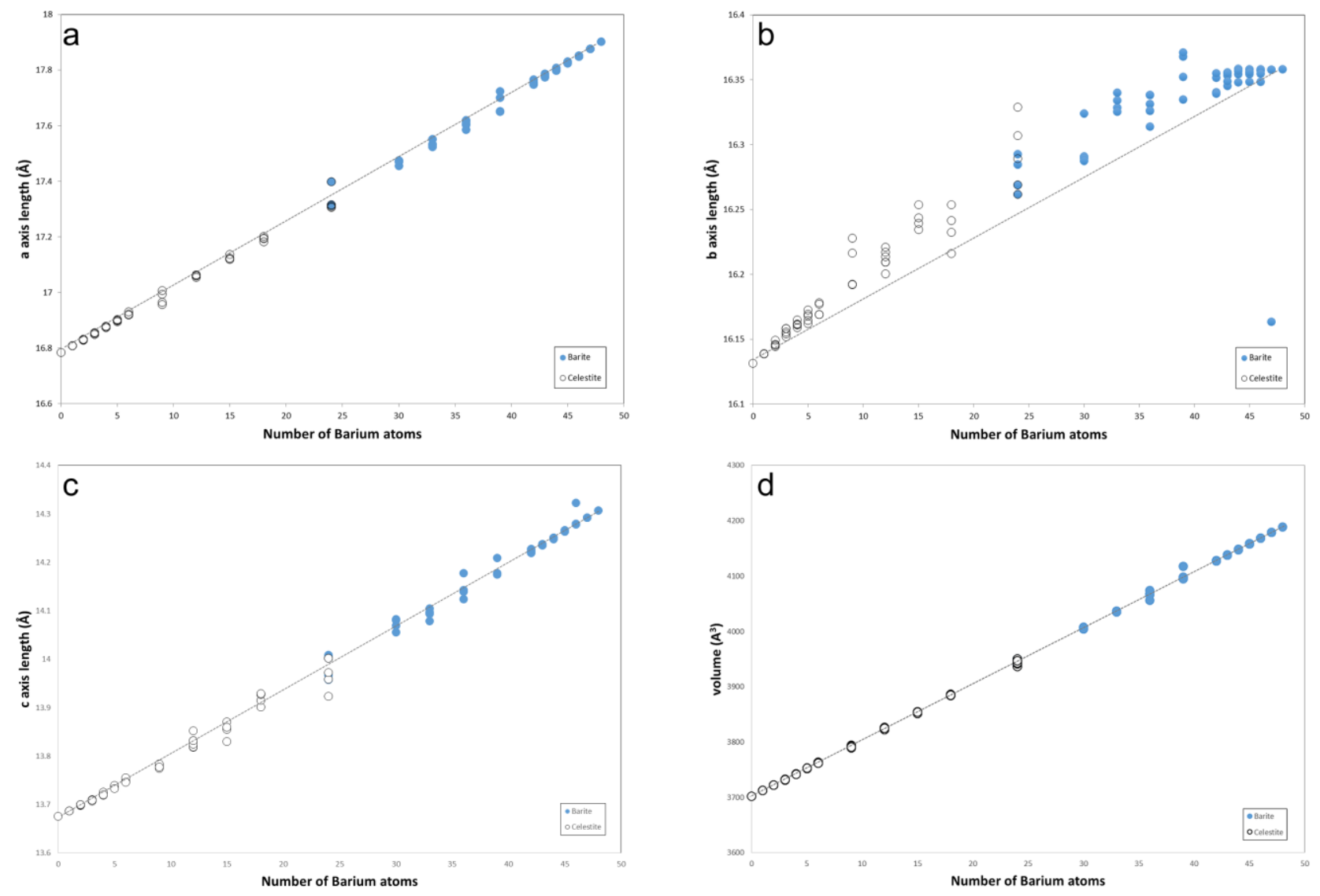

SFigure 8. Simulation results of lattice parameters and volume versus number of barium ions a) $a$ axis b) $b$ axis c) $c$ axis and d) Volume 


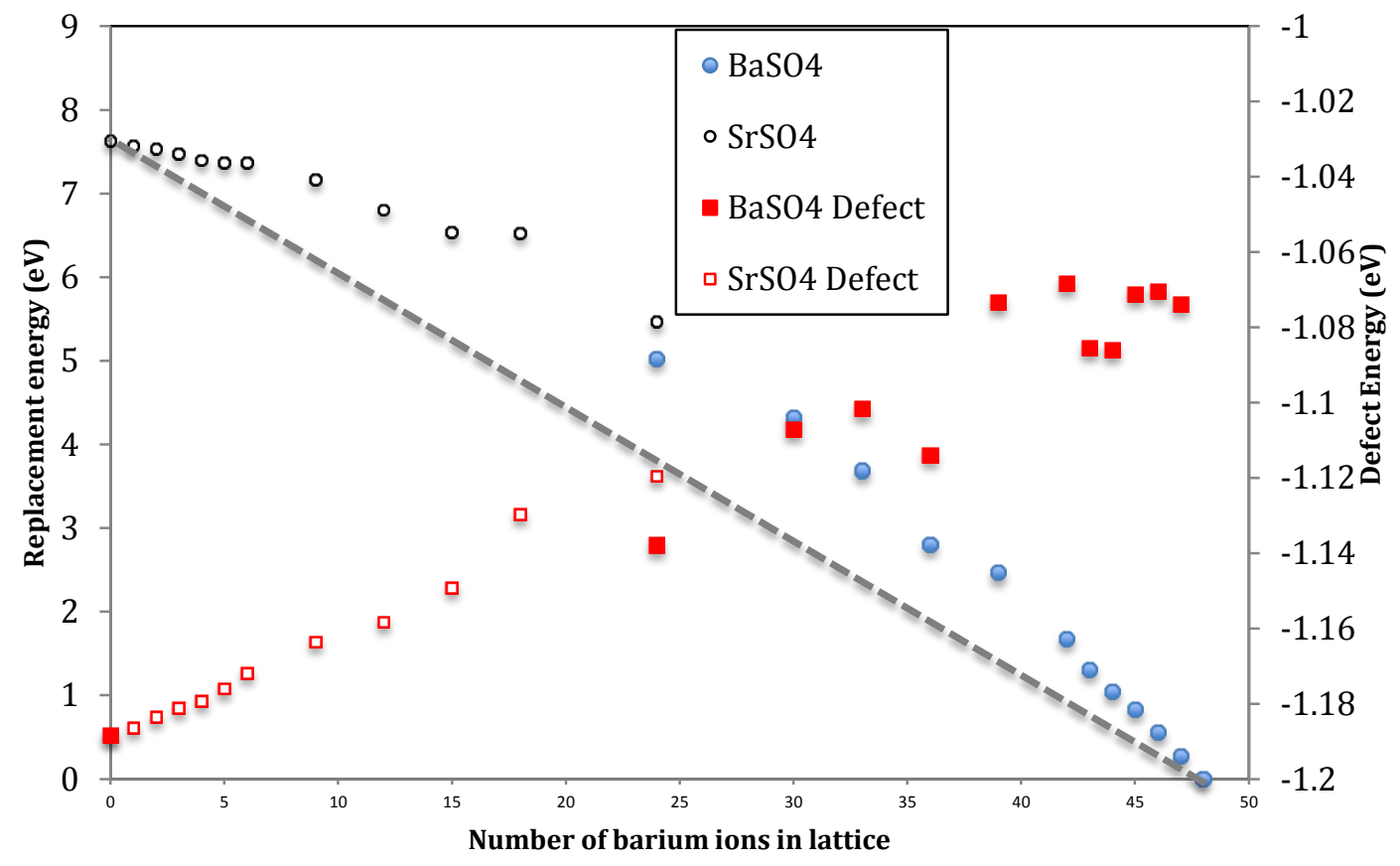

SFigure 9. Average replacement energy and average defect energy versus number of barium ions in the lattice (0 means pure $\left.\mathrm{SrSO}_{4}\right)$. Filled symbols represent data calculated from substituting into barite, open symbols represent data calculated from substituting into celestite.

The energetics of substitution in this work have been calculated in vacuo and the experiments are in aqueous environments meaning the results could be energetically favorable in water. 


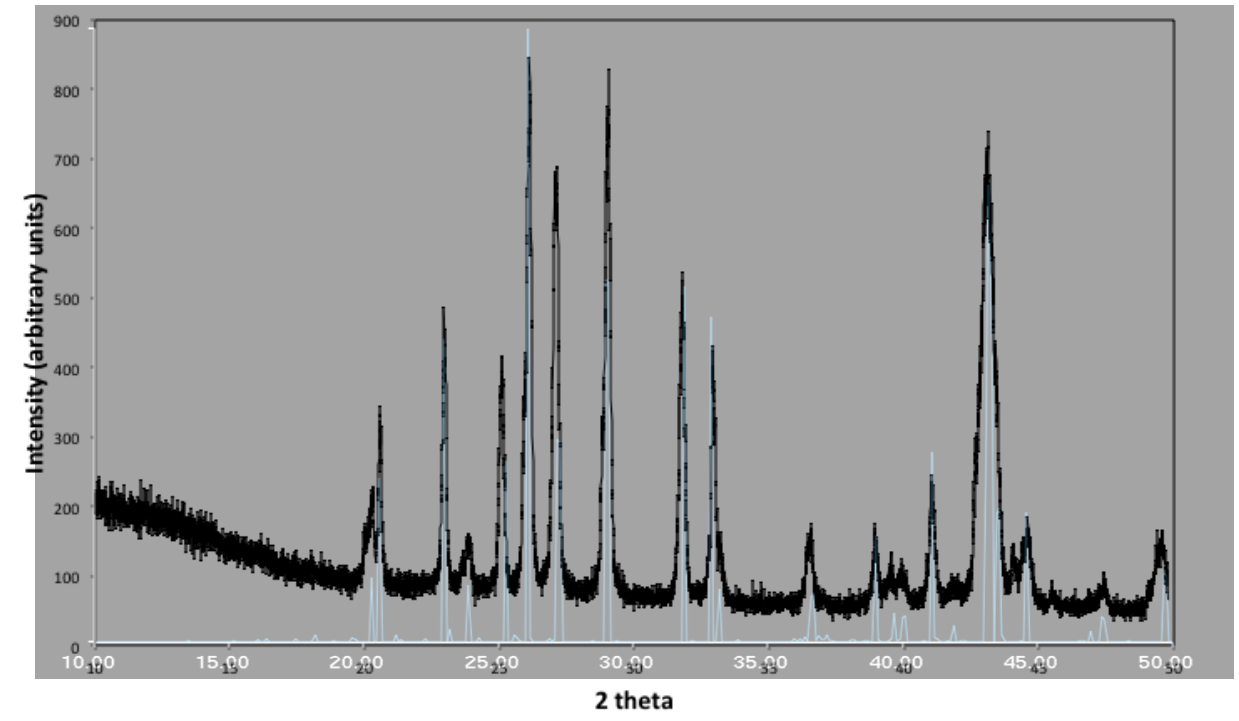

SFigure 10. Simulated XRD $\left(\mathrm{Ba}_{33} \mathrm{Sr}_{15} \mathrm{SO}_{4}\right)$ and experimental XRD (for solids obtained from SSW) superimposed on each other. 